\title{
Ringworm in dogs
}

\author{
Ahmed Hesham Ahmed Farag Kandil, Samar Magdy Mohamed Atwa
}

Department of Internal Medicine and Infectious Diseases, faculty of Veterinary Medicine, Mansoura University

\section{ARTICLE HISTORY}

Received: 15.02.2021

Revised: 24.03.2021

Accepted: 25.03.2021

Address correspondence Ahmed Kandil

\section{ABSTRACT}

Dermatophytosis is a common self-limiting zoonotic fungal disease which caused by infection of certain types of dermatophyte that fungi of 3 fundamental genera specifically, Microsporum, Trichophyton and epidermophyton. The present study presents the clinical presentation of ringworm in dogs

Keywords: Fungi, ringworm, dogs.

\section{INTRODUCTION}

Dermatophytosis is a common self-limiting zoonotic fungal disease which caused by infection of certain types of dermatophyte $[1,2]$ that fungi of 3 fundamental genera specifically, Microsporum, Trichophyton and epidermophyton.

We will not wait its self-limiting cause it Causes economic losses. That disease spread fastly amoung all existing dogs and may be owners. And take an extended time in treatment.

Dermatophytosis is generally called as ringworm. And that is visibly portrayed by multi-focal alopecia and hulls on the skin with a selected formation $[3,4]$.

This infection has gained specific attention publicly health, because it distributed all over the world. After touching the contaminated items or specimens, similar to hair, soil or outside on the epidermal layer of diseased animals, infection of dermatophytosis in human happen [5].

We have noted an increase in annual cases of dermatophytosis in all species humans and animals particularly dogs and cats [6] Scientists noted that dermatophytosis's cases are more predominant in little and female cat than male cat.

In Indonesia, much researches has been done on dogs shows that : in Yogyakarta, Indonesia (34\%) of dogs were positive of dermatophytosis [1] While that number ranges from $20 \%$ to $30 \%$ in Europe [7].

The results of different investigations recognized that main species causing dermatophytosis in small animals is microsporum-canis in $81.8 \%$ to $97 \%$ of total cases $[1,2,6]$.

The occurrence of zoonotic dermatophytes, in dogs and cats and human compared with different species, $M$. canis was the most awesome (60\%) [8] We can modulate the immune-response by giving significant level of cortisol throughout along period.

Other researchers noticed that these worms could also be present in up to $33 \%$ of adult dogs. So the aim of that scientific-review is making the reader more experience and have the great background about one among the foremost important infectious disease which is termed the ringworms and know that: what's that disease, why it's dangerous, mode of action and the way to treatment.

\section{Synonyms}

ringworm , dermatophytosis , trichophytosis , dermatomycosis Ringworm, which is somewhat misleading, is the common name for this disease, since it is not an infection caused by a worm, and the infected areas are not necessarily round-shaped. A specialist community known as dermatophytes belongs to the fungi responsible for ringworm infections, thus the medical term for that disease is dermatophytosis [9].

\section{Definition}

Ringworm is a common infection of the skin caused by fungi. They affect human and animal superficial keratinized tissue (hair, nail, and skin) caused by dermatophytes' invasion of keratinized epithelial cells and hair fibres. It has a distinctive character that is alopecia with or without crust formation in circular scaly areas [3].

This disease is a widespread disease that exists in all animals and humans as well.

\section{Etiology}

Over than 20 unique types of dermatophytes have been accounted for to cause clinical illness in the canines as well as cats. However most normally secluded microorganisms are the Microsporum canis,M. gypseum and the Trichophyton mentagrophytes.the Infection happens by directtransmission of infective spores to the defenseless host.the supplies of disease for the two individuals and creatures incorporate polluted conditions and the objects,animals with subclinical or clinical contaminations, and the creatures that are mechanical transporters of the spores on their hair coat. The illness commonness is obscure as this isn "t a reportable infection in many nations on the planet .

There are the outcome from the trial. the complete canines in this trial is 285 dog exhaustive portrayal, an aggregate of 285 (78.7\%) examples were discovered to be 
decidedly tainted with various dermatophytes among that pervasiveness of disease was the most elevated in felines $(158,55.5 \%)$ than canines $(108,37.8 \%)$. The settlements of Microsporum canis and Microsporum gypseum were wooly elevated mycelium, light to ruddy earthy colored pigmentation. The microscopical examination uncovered the presence of very much created macroconidia with 6-12 septa and little microconidia with followed appearance. The confines of Microsporum spp. demonstrated rich development on rice grain medium with ruddy to orange pigmentation.

Table 1. Prevalence of dermatophytosis in dogs.

$\begin{array}{lll}\text { Fungal species } & \text { No. } & \% \\ \text { M. canis } & 66 & 61.1 \\ \text { M. gypseum } & 24 & 22.2 \\ T . & 18 & 16.7 \\ \text { mentagrophytes } & & \\ \text { T. rubrum } & 0 & 0 \\ \text { Total } & 108 & 100.0\end{array}$

T.mentagrophytes = Trychophyton mentagrophytes

M.canis = Microsporum canis

M.gysperum = Mentagrophytes gysperum

T.rubrum = Trychophyton rubrum

\section{Epidemiology}

The ringworm is infectious and, by direct contact with the fungus, transmission occurs. It can pass through direct contact with an infected animal or human, or through the handling or touching of contaminated items or contaminated surfaces.

On combs, brushes, food dishes, furniture, bedding, carpet, or other environmental surfaces, the fungal spores may remain dormant for several months (reportedly up to 18 months) [9].

An infection does not always result from contact with the ringworm fungus.

The amount of environmental contamination, such as the age of an exposed person or animal, is a significant factor in the development of ringworm infection. In general, healthy adult humans are immune to infection unless there is a skin break, such as a scratch. Older people, young children, and adults with deficiencies in the immune system or sensitivities to the skin are particularly vulnerable to ringworm infection.

Infection is not assured by mere exposure to dermatophyte spores. The un-known, but the "critical mass" of the spores must touch the susceptible host. The host defence mechanisms, including mechanical elimination, competition with natural bacterial and fungal flora, exposure to epidermal lipid fungistatic properties, low skin surface moisture and acquired host immunity, must be prevented by spores. Infection-friendly factors include any pre-existing disease that will cause surface humidity to increase, because skin microtrauma and/or weaken host immune surveillance.
Recovery from infection relies on an immune response mediated by a competent cell.

\section{Mode of action}

We can say that the "Dermatophytes" may live on the outer surface of cornified layers of the skin. Some fungi have the capacity of sticking to particular host which result from many mechanisms and host factors. These factors also contain the capacity of fungi to adapt to the body. The Type of infection which occures naturally by deposition of viable Arthrospres or hyphae on surface of susceptible individual. After vaccination "injection into the skin of the host" during suitable conditions, the chance is valid for the infection to progress and this occures by subsequent stages [10] [11].

\section{Adherence}

After overcoming the hurdles such as: (ultraviolet light, temperature and moisture variation) and competing with traditional plants and "Sphingosines"which produced by Keratinocytes.Thus, the fatty acids produced by the sebaceous glands. The Arthroconidia which considered as infectious element adhere to keratinized tissue [12].

The growth of the hyphae and arthroconidial germination continues radially in various directions. We understood only alittle about the elements that mediate dermatophytes's Adherence. However, it been established that dermatophytic-discharged proteases could encourage or possibly be important for effectual Adherence $[13,14]$.

Trichophyton rubrum capacity to adhere to epithelial cells has been ascribed to specific adhesins of carbohydrate, which prevents on the mitochondria's surface.During the Adherence stage, fibriller projection are seen in $T$. mentagrophytes, from a morphological point of view.

At the surface of skin, fungal arthroconidia are connected to keratinocytes and to all other by the sparse and long fibrils.But in the inner layer of skin, there are short and thin appendices covering the whole surface of recently formed arthroconidia. Which begin to disappear as a huge contact zone is set up between the skin tissue and conidia [13].

\section{Penetration}

Arsenal promotes the dermatophytes to be able to digest the keratin network into assimilable oligopeptides or amino acids. When it's just founded, the spores begins germination. After that, it penetrates the corneum faster than desquamation.

The Penetration strings along with dermatophytes secreting Multiple Serine-Subtilisins and Metalloendoproteases(Fungalysins) formerly which we named (Keratinases) which confined its existence in the dermatophytes.

Keratinases are related directly to pathogeicity and this relationship is established by (Vai et al).Although we know low information about hydrolases like;lipases and ceramidase were Formed by these fungi. 
The mucolytic enzymes are responsible for the mechanism, this mechanism is involved in the penetration and the source of Nutrition to the fungi is unknown.The Keratenolytic Dermatophytic Proteases stop performance unless diulified Bridges were decreased in wards the compact protein network which comprise keratinised tissue.

Lately, we discovered that it depends on a Sulfite efflux pump which incoded by Ss1 gene, the sulphite is secreted by this transportation which permits sulfitolysis of the protiens, making them available for proteases, and at the same time it performs as a possible way for detoxification.And this considered as a future aim of creating anti-fungal treatment.

The production of protease on T.rubrumis very host specific which show reduced physiological activity when becoming on their favored host $[10,15]$. And this is able to clarify the well-established anthroppohization of those species.Identical finding on the connection between low protease profile of T.rubrum isolates and chronicity, has been announced by Ranganathan.

The mannans of fungal inside the cell wall of dermatophyte have immuno-inhibitory impact and cell wall mannans of T.rubrum (TRM) appear to be involved in phenomenon of immuno-suppression, restraining lymphoproliferative reaction of mono-nuclear leukocytes in response to many antigens (Whether it is dermatophyte or not) and mitogens.

In any case, during persistent infections, specific suppressor of T-cells are eventually activated, TRM's target cell activity view to be monocytes rather than lymphocytes. The Trichophyton rubrum mannans have also the ability to decrease the multiplication rate of kiratinocyte. And it can do that by two-ways, directly or by alternation of the lymphocyte function, contributing greatly to the chronicity of infection of T.rubrum.

However there's no homogenicity in substrate preference with all dermatophytes species which pervade the stratum corneum of skin but we observed large variation in their ability to pervade hair \&nail [12] [13] [16] [17]. Susceptible host: This disease is a widespread disease that exists in all animals and humans as well.

\section{Factor affecting susceptibility}

Any animal is susceptible to that disease (any age, any sex or even any breed), while that infection tend to be wide spread in young animals, diseased animals, debilitated and the old one.

Table 2. .Sex-wise incidence of dermatophytes in dogs.

$\begin{array}{lll}\text { Host } & \text { Dogs } & \\ \text { Positive } & \begin{array}{l}\text { Number } \\ \text { of cases }\end{array} & \\ \text { cases } & \text { of } \\ \text { Male } & 63 & 58.3 \\ \text { female } & 45 & 41.7\end{array}$

The susceptibility to the infection increases, when animal has another disease. The cats which have feline-immune deficiency virus are 3 times more susceptibility to infection than other normal cats.

Veterinarians thought that the long haired animal is more susceptible to infection. I think that's right and this may due to the truth that long hairs animals trap spores and even we called it "fungs friendly" climate or because of some hereditary factors.

But we found some studies which use experimental models for dermatophytosis recognized that: the infection is difficulties in the establishment when they allow to the cat for grooming. So we may consider that grooming as hostdefense mechanism but still under recognized.The rate of infection in male dogs (58.3\%) is higher than the infection rate in bitches $(41.7 \%)$ (8). So that we can say that these factor is the disease is more common where animals are housed in close proximity to teach other for long period

1. Animals under stress like environmental stress, pregnant animals

2. Animals with old age or young animals

3. Animals in poor condition

4. Animals treated with immune- suppressed drugs

5. Pathogenis

- Ring worm fungi attack chiefly keratinized tissue. Resulting in autolysis of the fiber structure. Incubation period of (M. canis) may be from 1 to 4 weeks. this causes breaking off of hair and alopecia , as well as allergic dermatitis and dry crust formation [18].

- Dermatophytosis are considered strict aerobes. And the fungi die under the outside layer in the middle of most sores. Leaving just the periphery active.

- The immunity is specific to the fungal species concerned and lasts for up to 2 years.

\section{Symptoms and morbidity rate}

The ringworms in dogs frequently appear as some combination of the following

*Alopecia (fall of hair) which might be round or patchy

*a weak and broken hair and a weak hair coat

*ulcerated or reddened skin

*the presence of scales in the head

*blackened skin

*there may be some itching and maybe not

Some time, people classify the dogs as silent carrier, and that means that dogs can still pass that disease to all species (human and animals). While it has no visible signs of that condition [19].

There are some breeds of dog have more susceptibility to dermatophytosis. There are several cases reported of the Yorkshire terrier dogs recognized as being more susceptible to the superficial and subcutaneous dermatophytosis. The most common case due to $\mathrm{m}$. Canis [16, 20-22]. 
There is one important study says that 13 from 55 and that presented $(23.6 \%)$ of dogs which suffering from dermatophytosis were yorkshire terrier dogs. While there is another study says 10 from 27 and that presented (37\%) of dogs were also yorkshire terrier dogs. There are other breeds also appear more susceptibility to dermatophytosis like working\&hunting breeds ( and that like German short haired, pointers, fox terrier, Labrador, jack russell terrier, German shepherd, beagle and belgian groenendael), specifically m.persicolor \& m.gypseum and that maybe because increase contact with contaminated soil $[23,24]$.

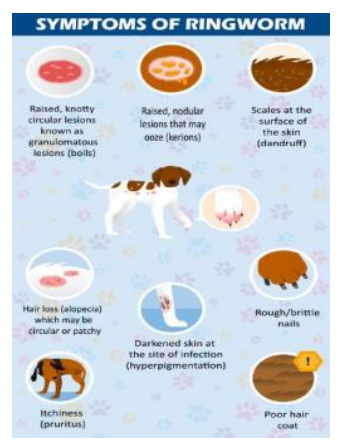

Figure 1: Show clinical signs of ringworm in dogs [25].

\section{Diagnosis}

\section{Field diagnosis}

This relies on the clinical symptoms of typical lesions. We may also use the UV lamp called the wood lamp, but not all cases of $\mathrm{M}$. canis can fluoresce under a wood lamp, and neither T. mentagrophytes nor M. gypseum fluoresce. Some skin ointments and other materials are also fluorescent and can offer a false positive outcome. Although this method is appropriate as a rapid screening method in multi-animal facilities in some cases, diagnosis made on Wood's lamp assessment is not always accurate [26].

\section{Laboratory diagnosis}

Fungal culture of a sample of hair or skin cells is the most accurate approach for the diagnosis of ringworm in dogs. A positive culture may often be confirmed within a few days, but the fungal spores may be slow to develop in certain instances, and the results of the culture can take up to four weeks. Hence, for at least a month, a suspected sample may not be considered negative.

The causes of hair loss in dogs are various. Your veterinarian can suggest additional testing before making a ringworm diagnosis to rule out any of these causes [27].

\section{Differential diagnosis}

Skin scraping examination may be required to distinguish ringworm from mycotic dermatitis, manage and other cutaneous infections, so we can't use only clinical signs for diagnosis [9].

\section{Treatment}

We should understand that the majority of cases of this illness are self-limiting.Owing to the infectious and zoonotic nature (transmissible to humans) of certain types of ringworm, quarantine procedures should be considered.The primary purpose of the treatment is to reduce the spread to other animals and humans.There are two lines for treatment :

\section{Topical treatment}

In mild cases, only topical therapy may be sufficient to speed up healing and minimise the risk that the disease may spread to other animals or individuals. Shaving a long coat for the pet can help topical medicines penetrate the skin. Lime sulphur dips, enilconazole rinses, and miconazole shampoos are options. Dichlorophen was used every day for 5 days. Topical application of iodine (2\% lugol iodine, $1 \%$ iodophors or $2 \%$ iodine tinicture) 3-4 times daily for 7 days [27].

\section{Systemic treatment}

An oral anti-fungal medication is needed for successful treatment. Griseofulvin is the most commonly used medication for this reason, although newer medications such as itraconazole or terbinafine (Lamasil) are used more often and are also favoured because they have less side effects.

Individual dogs' reaction to treatment varies and the disease can recur if therapy is stopped too soon. Treatment typically lasts for a minimum of 6 weeks and requires even longer counselling in some cases. At the start of therapy, ringworm cultures are taken regularly to assess if your pet is still infected [28].

Usually, two consecutive negative cultures of ringworm suggest that your pet has been treated adequately, after which point care may be stopped.

If an underlying disorder (e.g., malnutrition, immunosuppressive drug administration, etc.) is found to play a role in the ringworm development of the dog, it should also be discussed. But we should still give medication, such as zylexis, to improve immunity $[19,28]$.

\section{Prevention and control of the disease}

To prevent spreading of the infection to others, you should:

*Isolate the infected animal which undergoing treatment

*Wash your clothes and skin after handling with sick animal

*When you deal with infected animal, wear clean gloves

*Decontaminate your house, clean floors and surfaces with effective disinfectant

(The best choice in that time is dilute bleach solution) [9, $27,28]$.

\section{Control of the infection}

Before anything you should isolate the diseased dog from the other and contaminate everything he used or touched You can, by dilute chlorine bleach using the dilution of half liter of chlorine bleach with 4 liter of water or a dilution from 1:10 to $1: 100$, kill contagious fungal spores.Or you can 
use formaldehyde 2\% [29]. Don't forget that: Expose the animal to sunlight daily, play a great role.

That's a few important points which regarding dog ringworm, which every dog owner should know

- Dermatophytosis affects chiefly immunocompromised dogs and also the dogs under stress, such as kittens, pregnant dogs, old dogs etc.

- That disease easily spread among dogs and humans by two 2 method: (i) direct contact with diseased dogs. (ii) in- direct contact with contaminated items such as, the toys, combs, towels etc [9]

- Dermatophytosis's treatment takes extended period (30-45 days).

- During treatment of your dog, you should give your dog a good quality and quantity of the food.

- Avoid bathing your dog repeatedly due to the continuous humidity on its skin helps in spread of that diseases.

- Shaving the hair of your infected dog aids in better treatment.

- Expose the animal to sunlight daily, play a great role in treatment [28].

Practical application in the clinic

When I trained in one of the most popular pet's clinic in Egypt There is a bulldog dog brought to the clinic. She was suffering from hair loss with patches on her body devoid of hair. In this time the doctor examined her and used the UV lamp which called wood's lamp in order to closely examin its skin.

We found fluorescence of these patches and this is a rapid technique to diagnose a very common disease which called the ringworms. The doctor tell to the owner that this disease is zoonotic disease and we started giving her the vacderm 3injections with a gap of 12 days each. Along with that we gave her a local cream "miconaz" twice daily for a span of 21 days and zylexis to raise her immune system. And advice the owner to let the dog sit in the sun daily especially after sunrise and before sunset for half an hour.

The sun plays vital role in treatment of this disease.After finishing the course of treatment for about 45 days. Her hair have grown again beautifully.

\section{REFERENCES}

[1] Indarjulianto $S$, Yanuartono $Y$, Widyarini S, Raharjo S, Purnamaningsih $H_{\text {, }}$ Nururrozi A, et al. Jurnal Veteriner. 2017;18:207.

[2] Ivaskiene M, Matusevicius AP, Grigonis A, Zamokas G, Babickaite L. Efficacy of Topical Therapy with Newly Developed Terbinafine and Econazole Formulations in the Treatment of Dermatophytosis in Cats. Polish Journal of Veterinary Sciences. 2016;19:535-43.

[3] Haggag Y, Samaha H, Nossair M, Mohammad A. Prevalence of Dermatophytosis in some animals and Human in Behera Province, Egypt. Alexandria Journal of Veterinary Sciences. 2017;53:64.

[4] Tater KC. An approach to pruritus. BSAVA Manual of Canine and Feline Dermatology: British Small Animal Veterinary Association; 2012. p. 3745.

[5] Rashidian S, Falahati M, Kordbacheh P, Mahmoudi M, Safara M, Tafti HS, et al. A study on etiologic agents and clinical manifestations of dermatophytosis in Yazd, Iran. Current medical mycology. 2015;1:20.
[6] Frymus T, Gruffydd-Jones T, Pennisi MG, Addie D, Belák S, BoucrautBaralon C, et al. Dermatophytosis in Cats. Journal of Feline Medicine and Surgery. 2013;15:598-604.

[7] Murmu S, Debnath C, Pramanik AK, Mitra T, Jana S, Dey S, et al. Detection and characterization of zoonotic dermatophytes from dogs and cats in and around Kolkata. Veterinary World. 2015;8:1078-82.

[8] Cafarchia C, latta R, Latrofa MS, Gräser Y, Otranto D. Molecular epidemiology, phylogeny and evolution of dermatophytes. Infection, Genetics and Evolution. 2013;20:336-51.

[9] Skarda RT. Techniques of local analgesia in ruminants and swine. Vet Clin North Am Food Anim Pract. 1986;2:621-63.

[10] Rippon JW. The Changing Epidemiology and Emerging Patterns of Dermatophyte Species. Current Topics in Medical Mycology: Springer New York; 1985. p. 208-34.

[11] Samdani AJ. Dermatophyte growth and degradation of human stratum corneum in vitro (pathogenesis of dermatophytosis). JAMC: Journal of Ayub Medical College, Abbottabad. 2005;17:19.

[12] Tainwala R, Sharma YK. Pathogenesis of dermatophytoses. Indian journal of dermatology. 2011;56:259.

[13] Vermout S, Tabart J, Baldo A, Mathy A, Losson B, Mignon B. Pathogenesis of Dermatophytosis. Mycopathologia. 2008;166:267-75.

[14] Aljabre SHM, Richardson MD, Scott EM, Rashid A, Shankland GS. Adherence of arthroconidia and germlings of anthropophilic and zoophilic varieties of Trichophyton mentagrophytes to human corneocytes as an early event in the pathogenesis of dermatophytosis. Clinical and Experimental Dermatology. 1993;18:231-5

[15] Stelzner A. John Willard Rippon, Medical Mycology-The Pathogenic Fung and the Pathogenic Actinomycetes (Third Edition). IX + 797 S., 482 Abb., 58 Tab. Philadelphia-London-Toronto-Montreal-Sydney-Tokyo 1988. W. B. Saunders Company-Harcourt Brace Jovanovich Inc. $f 50.00$ ISBN: 07216-2444-8. Journal of Basic Microbiology. 1990;30:463-.

[16] Dahl MV. Dermatophytosis and the immune response. Journal of the American Academy of Dermatology. 1994;31:S34-S41.

[17] Chander J. Textbook of medical mycology: JP Medical Ltd; 2017.

[18] Moriello KA. Zoonotic skin diseases of dogs and cats. Animal Health Research Reviews. 2003;4:157-68.

[19]

. 2018 Published: September 08, 2008.

[20] Cerundolo R. Generalized Microsporum canis dermatophytosis in six Yorkshire terrier dogs. Veterinary Dermatology. 2004;15:181-7.

[21] Cafarchia C, Romito D, Sasanelli M, Lia R, Capelli G, Otranto D. The epidemiology of canine and feline dermatophytoses in southern Italy. Zur Epidemiologie der Dermatophytose von Hund und Katze im Suden Italiens. Mycoses. 2004;47:508-13.

[22] Yokoi S, Sekiguchi M, Kano R, Kobayashi T. Dermatophytosis Caused by Trichophyton rubrum Infection in a Dog. The Japanese Journal of Veterinary Dermatology. 2010;16:211-5.

[23] Guaguere E, Muller A, Degorce-Rubiales F. FC-43 Feline demodicosis: a retrospective study of 12 cases. Veterinary Dermatology. 2004;15:34-

[24] Carlotti, Bensignor. Dermatophytosis due toMicrosporum persicolor(13 cases) orMicrosporum gypseum(20 cases) in dogs. Veterinary Dermatology. 1999;10:17-27.

[25] Canna Pet. Ringworm in Dogs: Causes, Signs, \& Treatment. August 8, 2017.

[26] Asawanonda P, Taylor CR. Wood's light in dermatology. International Journal of Dermatology. 1999;38:801-7.

[27] Yaksh TL. Pharmacology of spinal adrenergic systems which modulate spinal nociceptive processing. Pharmacol Biochem Behav. 1985;22:84558.

[28] yousef AA. https://creature-companions.in/cat-ringworm-infectiousskin-disease-in-cats.

[29] Kinjavdekar P, Singh GR, Amarpal, Aithal HP, Pawde AM. Clinicophysiological effects of spinally administered ketamine and its combination with xylazine and medetomidine in healthy goats. Vet Res Commun. 2007;31:847-61. 